\title{
Uncommon Association of Tuberculous Dactylitis and Cutaneous Polyarteritis Nodosa in a Hemodialysis Patient
}

\author{
Picha Yincharoen $^{a} \quad$ Nattachai Srisawat ${ }^{\text {b, c }}$ \\ aDivision of Nephrology, Bhumirajanagarindra Kidney Institute Hospital, \\ Bangkok, Thailand; 'bivision of Nephrology, Department of Medicine, Faculty of \\ Medicine, Chulalongkorn University, and King Chulalongkorn Memorial Hospital, \\ Bangkok, Thailand; 'Center for Critical Care Nephrology, The CRISMA Center, Department \\ of Critical Care Medicine, University of Pittsburgh School of Medicine, Pittsburgh, PA, USA
}

\section{Keywords}

Tuberculous osteomyelitis · Dactylitis · Hemodialysis · Mycobacterium tuberculosis · Abscess

\begin{abstract}
Tuberculous dactylitis, defined as the infection of the metacarpals, metatarsals, or phalanges of the hands or feet, is an uncommon manifestation of extrapulmonary tuberculosis. It is mainly seen in pediatric populations, especially those less than 6 years old. Here, we present a case of tuberculous dactylitis in an elderly patient with end-stage renal disease on hemodialysis. Moreover, our patient also developed generalized nodular lesions during hospitalization. Skin biopsy was consistent with cutaneous polyarteritis nodosa, which is a rare association with tuberculosis. She was treated with antituberculous drugs with significant improvement in both cutaneous nodules and dactylitic lesions.
\end{abstract}




\section{Introduction}

Tuberculosis (TB) is one of the major causes of significant morbidity and mortality, especially in developing countries. The estimated prevalence of the disease in Asia and Africa was 9 million cases in 2013 [1].

TB manifestations can be categorized mainly into pulmonary and extrapulmonary manifestations. Extrapulmonary TB can occur either isolated or secondary to primary foci in the lung. Skeletal TB refers to TB involvement of bones and/or joints, and it accounts for $10-35 \%$ of extrapulmonary TB cases. The most common form of skeletal TB is a disease of the spine (Pott's disease), which comprises approximately 50\% of cases. The second most common form is tuberculous arthritis, involving weight-bearing joints, i.e., the hip and the knee [2], followed by extraspinal tuberculous osteomyelitis. There were reports of tuberculous osteomyelitis involving the maxilla, sternum, ulna, femur, humerus, and shoulder joint with fracture and/or dislocation [3].

Tuberculous infection of metacarpals, metatarsals, or phalanges of the hands or feet is specifically defined as tuberculous dactylitis. Eighty-five percent of patients with tuberculous dactylitis are younger than 6 years of age, and it is quite a rare manifestation in adults [4].

\section{Case Presentation}

A 63-year-old Thai woman, with a history of coronary artery disease status post bypass surgery and intracardiac device as well as end-stage renal disease on regular hemodialysis 2 sessions per week for the past 1.5 years, presented to our hospital with an abscess in the right hand for 4 weeks and failed antibiotic treatment at an outpatient hospital.

Four weeks prior to hospitalization, she started to notice right hand pain while she was wearing her shoes. She could not recall any significant trauma or injury. She then went to an outpatient hospital and was found to have developed an abscess in the right hand. She underwent needle aspiration, and pus was sent for bacterial culture, which was negative. She was treated with oral antibiotics for 2 weeks, but her abscess was getting worse, so she presented to our hospital for further evaluation. She denied fever, malaise, and significant weight loss.

A physical examination revealed tenderness, redness, swelling over the right hand around the punctured site, and a restricted range of movements. There was purulent discharge from the surgical site. Laboratory tests showed WBC $20,200 / \mathrm{mL}$ (neutrophils $90 \%$, lymphocytes $4 \%$, monocytes 5\%), ESR $59 \mathrm{~mm} / \mathrm{h}$, and hs-CRP $6.11 \mathrm{mg} / \mathrm{dL}$. X-ray of her right hand demonstrated an osteolytic lesion and a fracture of the second metacarpal bone of the right hand (Fig. 1). She was diagnosed with pathological fracture and osteomyelitis at the second right metacarpal bone.

Incision and drainage were performed, and pus was sent for bacterial and mycobacterial cultures. She was started on broad-spectrum antibiotics for 14 days, then deescalated to levofloxacin, with partial improvement. She continued to have active drainage from her wound, requiring her to stay in hospital for daily wound care.

Four weeks after admission, she developed new-onset fever and scattered erythematous, painful nodular lesions on the bilateral upper and lower extremities. Skin biopsy was performed, and the pathology was consistent with cutaneous polyarteritis nodosa (cPAN). The antibiotics regimen was adjusted to be empiric for nontuberculous mycobacterial infection (imipenem, ethambutol, levofloxacin, and azithromycin) while the cultures were in process. 
Six weeks after admission, the initial pus culture turned out positive for Mycobacterium tuberculosis. The patient was diagnosed with tuberculous dactylitis, and was initiated on antituberculous treatment with a 4-drug regimen (isoniazid, rifampicin, pyrazinamide, and ethambutol). Her clinical signs and symptoms significantly improved, and she could be discharged home. At the 2-month follow-up visit, her dactylitic area continued to improve without further drainage and her nodular skin lesions were completely resolved. She was then transitioned to a maintenance regimen with isoniazid and rifampicin for 10 more months with monthly follow-up visits.

\section{Discussion}

TB infection is usually resulting from impaired host immunity and/or heavy exposure to the infection. There are many known risk factors for TB infection, e.g., age, malnutrition, HIV infection, diabetes mellitus, transplantation, malignancy, and low socioeconomic status [5]. Advanced chronic kidney disease is also one of the risk factors due to impaired immune function secondary to oxidative stress and inflammation, 25-hydroxyvitamin D deficiency, malnutrition, anemia, and hyperparathyroidism $[1,6]$. It seems clear that lymphopenia, suboptimal responses to mitogens, abnormal cytokine gene expression, and abnormal IL-2R expression are seen in a number of dialysis patients [7].

The incidence of TB among patients with advanced chronic kidney disease on dialysis is quite varied worldwide, ranging from 1.6 to 28\% [8]. It was found to be 6-25 times higher than in the general population, with mortality rates ranging from 17 to $75 \%$ [6]. Ates et al. [8] reported that the median time between initiation of dialysis and diagnosis of TB was 11 months, which is consistent with several studies showing a high incidence of TB in the first year of dialysis attributable to poor health in general and compromised host immunity.

In Thailand, there was a report in 2005 showing that the incidence of TB was 204 per 100,000 population and the mortality rate was 19 per 100,000 population [9]. However, to our knowledge, the incidence of TB infection specifically among dialysis patients has never been reported.

Contrary to other populations, extrapulmonary TB was found to be more common than pulmonary TB among dialysis patients, occurring in approximately 60-80\% [10]. Skeletal (or osteoarticular) TB is one of the extrapulmonary TB manifestations, and it was reported to account for $12.5 \%$ of extrapulmonary TB cases among hemodialysis patients [11]. Theoretically, it is often the result of hematogenous spread primarily from the lung; however, only $1 / 3$ of patients with skeletal TB are diagnosed with concomitant active pulmonary disease [4].

Tuberculous osteomyelitis was found to be the third most common form of skeletal TB in Thailand, occurring in approximately $10.1 \%$ of cases [9], but TB osteomyelitis of the metacarpal bone (so-called tuberculous dactylitis), as in our patient, is quite uncommon. Moreover, to make a diagnosis of tuberculous osteomyelitis is challenging, since it requires a high index of suspicion because there are no specific radiographic findings. Typically, the onset of the disease is quite long and can slowly progress in several years. Patients may be presenting with pain and swelling at the infected area, or with advanced-stage presentations such as articular destruction or abscess formation [12], as in our patient. Monach et al. [13] reported that patients with TB osteomyelitis may present with low-grade fever and exhibit so-called cold abscesses that do not show local redness or warmth. Cold abscesses have been reported in patients with chromomycosis, TB, actinomycosis, Trichophyton rubrum infections, protothecosis, Trichophyton violaceum infections, Job's syndrome, EF-4 bacilli, Pseudomonas aeruginosa 
sepsis, Cowden's disease with immunodeficiency, sporotrichosis, blastomycosis, nocardiosis, histoplasmosis, cryptococcosis, and pseudallescheriasis. Coagulase-positive staphylococci causing multiple sweat gland abscesses (periporitis) are also a cause of cold abscesses [14].

The duration of treatment for TB osteomyelitis remains controversial; usually, at least 12 months of therapy are recommended. The use a 4-drug regimen (isoniazid, rifampicin, pyrazinamide, and ethambutol) for 2-4 months, followed by a 2-drug regimen of isoniazid and rifampicin for 6-10 months depending on the response, is advised. The dosages of the drugs are adjusted according to creatinine clearance or glomerular filtration rate. Our patient had significant improvement at the 2-month follow-up, so that we switched from the 4-drug regimen to isoniazid and rifampicin with a monthly follow-up.

Another interesting point in our case was that the patient also developed nodular skin lesions whose pathology was consistent with cPAN. cPAN is often idiopathic, with an association with other medical conditions in perhaps $40 \%$ of patients, including various infections, autoimmune diseases, and medications. Infections reported to be linked with cPAN are group A Streptococcus (most common), hepatitis B virus, hepatitis C virus, parvovirus B19, and rarely Mycobacterium tuberculosis infection [15]. The skin lesions of our patient had completely resolved after initiation of the antituberculosis treatment; thus, we could imply an association between these two conditions that are quite uncommon.

\section{Statement of Ethics}

The patient in this report has given her informed consent.

\section{Disclosure Statement}

The authors have no conflicts of interest to declare.

\section{References}

1 Romanowski K, Clark EG, Levin A, Cook VJ, Johnston JC. Tuberculosis and chronic kidney disease: an emerging global syndemic. Kidney Int. 2016 Jul;90(1):34-40.

2 Kayabasi H, Sit D, Kadiroglu AK, Kara IH, Yilmaz ME. The prevalence and the characteristics of tuberculosis patients undergoing chronic dialysis treatment: experience of a dialysis center in southeast Turkey. Ren Fail. 2008;30(5):513-9.

3 Birole U, Ranade A, Mone M. A Case Report of an Unusual Case of Tuberculous Osteomyelitis Causing Spontaneous Pathological Fracture of Humerus in a Middle Aged Female. J Orthop Case Rep. 2017 JanFeb;7(1):41-5.

4 Kushwaha RA, Kant S, Verma SK, Sanjay, Mehra S. Isolated metacarpal bone tuberculosis-a case report. Lung India. 2008 Jan;25(1):17-9.

5 Li SY, Chen TJ, Chung KW, Tsai LW, Yang WC, Chen JY, et al. Mycobacterium tuberculosis infection of endstage renal disease patients in Taiwan: a nationwide longitudinal study. Clin Microbiol Infect. 2011 Nov;17(11):1646-52.

6 Nakazawa Y, Nishino T, Mori A, Uramatsu T, Obata Y, Arai H, et al. Tuberculous osteomyelitis in the ulna of a patient undergoing hemodialysis. Intern Med. 2013;52(1):135-9.

7 Kelly CJ. T cell function in chronic renal failure and dialysis. Blood Purif. 1994;12(1):36-41.

8 Ates G, Yildiz T, Danis R, Akyildiz L, Erturk B, Beyazit H, et al. Incidence of tuberculosis disease and latent tuberculosis infection in patients with end stage renal disease in an endemic region. Ren Fail. 2010 Jan;32(1):91-5.

9 Muangchan C, Nilganuwong S. The study of clinical manifestation of osteoarticular tuberculosis in Siriraj Hospital, Thailand. J Med Assoc Thai. 2009 Mar;92 Suppl 2:S101-9. 
10 Abdelrahman M, Sinha AK, Karkar A. Tuberculosis in end-stage renal disease patients on hemodialysis. Hemodial Int. 2006 Oct;10(4):360-4.

11 Chou KJ, Fang HC, Bai KJ, Hwang SJ, Yang WC, Chung HM. Tuberculosis in maintenance dialysis patients. Nephron. 2001 Jun;88(2):138-43.

12 Lopes MJ, Furtado I, Brandão M, Farinha F. Tuberculous osteomyelitis of the wrist presenting as a monoarthritis syndrome. BMJ Case Rep. 2018 Feb;2018:bcr-2017-222136.

13 Monach PA, Daily JP, Rodriguez-Herrera G, Solomon DH. Tuberculous osteomyelitis presenting as shoulder pain. J Rheumatol. 2003 Apr;30(4):851-6.

14 Jackson R, Stephens L, Kelly AP. Cold subcutaneous abscesses. J Natl Med Assoc. 1990 Oct;82(10):733-6.

15 Imanishi H, Tsuruta D, Oshimo T, Sowa J, Mizuno N, Nakagawa K, et al. Cutaneous polyarteritis nodosa induced by Mycobacterium tuberculosis. J Dermatol. 2012 Aug;39(8):738-9.

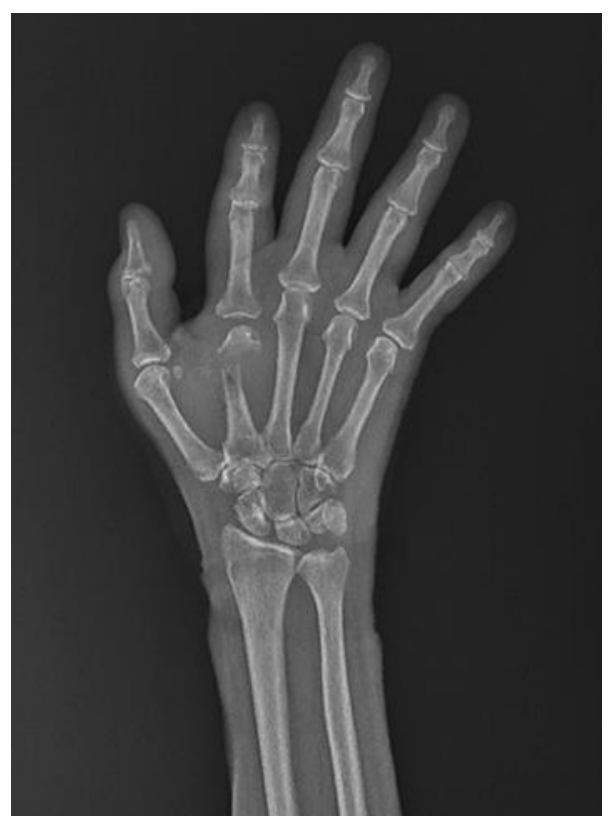

Fig. 1. X-ray image of the right hand (anteroposterior). 